\title{
A Relation between Nash Equilibria and Correlated Equilibria
}

Citation for published version (APA):

Peeters, R. J. A. P., Hendrickx, R. L. P., \& Potters, J. A. M. (2002). A Relation between Nash Equilibria and Correlated Equilibria. International Game Theory Review, 4, 405-414.

https://doi.org/10.1142/S021919890200077X

Document status and date:

Published: 01/01/2002

DOI:

10.1142/S021919890200077X

Document Version:

Publisher's PDF, also known as Version of record

\section{Please check the document version of this publication:}

- A submitted manuscript is the version of the article upon submission and before peer-review. There can be important differences between the submitted version and the official published version of record.

People interested in the research are advised to contact the author for the final version of the publication, or visit the DOI to the publisher's website.

- The final author version and the galley proof are versions of the publication after peer review.

- The final published version features the final layout of the paper including the volume, issue and page numbers.

Link to publication

\footnotetext{
General rights rights.

- You may freely distribute the URL identifying the publication in the public portal. please follow below link for the End User Agreement:

www.umlib.nl/taverne-license

Take down policy

If you believe that this document breaches copyright please contact us at:

repository@maastrichtuniversity.nl

providing details and we will investigate your claim.
}

Copyright and moral rights for the publications made accessible in the public portal are retained by the authors and/or other copyright owners and it is a condition of accessing publications that users recognise and abide by the legal requirements associated with these

- Users may download and print one copy of any publication from the public portal for the purpose of private study or research.

- You may not further distribute the material or use it for any profit-making activity or commercial gain

If the publication is distributed under the terms of Article $25 \mathrm{fa}$ of the Dutch Copyright Act, indicated by the "Taverne" license above, 
International Game Theory Review, Vol. 4, No. 4 (2002) 405-413

(C) World Scientific Publishing Company

\title{
A RELATION BETWEEN NASH EQUILIBRIA AND CORRELATED EQUILIBRIA
}

\author{
RUUD HENDRICKX \\ CentER and Department of Econometrics and OR, Tilburg University, \\ P.O. Box 90153, Tilburg, 5000 LE, The Netherlands \\ ruud@uvt.nl \\ RONALD PEETERS \\ Department of Economics, Universiteit Maastricht, \\ P.O. Box 616, Maastricht, 6200 MD, The Netherlands \\ JOS POTTERS \\ Department of Mathematics, Nijmegen University, \\ P.O. Box 9010, Nijmegen, 6500 GL, The Netherlands
}

\begin{abstract}
In this paper we investigate the set of correlated equilibria of bimatrix games. These equilibria are interesting, because they can result in outcome profiles that are not feasible as a result of Nash equilibria. After giving an example to illustrate the various concepts, we present a Projection Theorem which relates the two types of equilibria. Some lemmas are provided to clarify and extend this theorem.
\end{abstract}

Keywords: Nash equilibria; correlated equilibria; projection.

\section{Introduction}

In certain classes of strategic games the players have partially common interests and they may fear that "just playing a Nash equilibrium" does not do justice to the common interests. In such games it may be wise to introduce a cooperative pre-play meeting to coordinate the actions of the different players. The concept of correlated equilibrium is based on this idea (see Aumann $(1974,1987)$ ). It gives a method to coordinate the actions of the players before the game is played.

The idea is shown in the following: in the pre-play meeting the players agree upon a probability space (with a finite number of outcomes) and for each player a signalling function on the outcome space. When the game is played, a chance mechanism with the agreed probability measure determines an outcome (out of the reach of any player) and each player gets a signal according to his own signalling function. Next, each player chooses an action in his action space and the payoffs follow. The difference with playing the original strategic game is that the players can react to the signal they get. 
During the pre-play meeting the players transform the strategic game into a Bayesian game. A Bayesian equilibrium of the extended game is called a correlated equilibrium of the strategic game. Note that the signal is costless and is only used to coordinate the actions.

Osborne and Rubinstein (1994) show that if we start with an arbitrary Bayesian equilibrium, we can construct another one by choosing the set of all pure strategy profiles as the outcome space and for each player the signalling function assigning to every strategy profile his own component, which generates the same outcome as the original one. As the chance distribution over the set of pure strategy profiles is the only thing that matters, the players do not need fancy chance mechanisms and signalling functions. So, the set of correlated equilibria consists of all probability measures on the set of pure strategy profiles for which the reaction functions "following the advice you get" form a Bayesian equilibrium. Note that if "following the advice you get" is optimal for every player, the eventual payoffs to the players are fully determined by the agreed probability distribution. So, the discussion during the pre-play meeting is about the outcome.

In the recent literature, there has been some interest in the relations between Nash equilibria and correlated equilibria. For example, Evangelista and Raghavan (1996) prove that every extreme point of a maximal Nash set is also an extreme point of the set of correlated equilibria. The same result is shown in Canovas et al. (1999), who in addition pays special attention to completely mixed Nash equilibria.

Most of this literature, however, is not concerned with the corresponding payoffs to the players. In this paper, we provide a Projection Theorem for bimatrix games, which gives necessary and sufficient conditions for a projected Nash equilibrium to be a correlated equilibrium. An important feature of this theorem is that it yields a correlated equilibrium in which the payoffs to the players are higher than in the original Nash equilibrium. In case the original Nash equilibrium is completely mixed, some stronger results are obtained.

This paper is organised as follows. In Sec. 2, we introduce some basic notation and basic definitions. An introduction to correlated equilibria in bimatrix games is given in Sec. 3. After an example in Sec. 4, we state our Projection Theorem in Sec. 5 , followed by a number of lemmas.

\section{Notation and Basic Definitions}

Let $K \neq \emptyset$ be a finite set. Denote

$$
\Delta^{K}=\left\{x \in \mathbb{R}^{K} \mid \forall_{i \in K}: x_{i} \geq 0, \sum_{i \in K} x_{i}=1\right\}
$$

and for $x \in \mathbb{R}^{K}$, denote $C^{K}(x)=\left\{i \in K \mid x_{i} \neq 0\right\}$. Let $e^{i, K}$ denote the $i$ th canonical unit vector in $\mathbb{R}^{K}$ for every $i \in K$. 
For $x \in \mathbb{R}^{K}, x \geq 0$ and $S \varsubsetneqq C^{K}(x)$, define $x^{-S} \in \mathbb{R}^{K}$ by

$$
x_{i}^{-S}= \begin{cases}0 & \text { if } i \in S, \\ x_{i} \frac{\sum_{j \in K} x_{j}}{\sum_{j \in K \backslash S} x_{j}} & \text { if } i \in K \backslash S,\end{cases}
$$

for all $i \in K$.

Throughout this paper, let $M, N$ denote the finite action spaces of player 1 and 2 , respectively. We assume $|M|>1,|N|>1$. A bimatrix game is denoted by $(A, B)$ where $A, B \in \mathbb{R}^{M \times N}$ are the payoff matrices of player 1 and 2 , respectively. The expected payoff when the mixed strategy profile $(p, q) \in \Delta^{M} \times \Delta^{N}$ is played equals $p^{\top} A q$ for player 1 and $p^{\top} B q$ for player 2 .

For $D \in \mathbb{R}^{M \times N}$, denote by $D_{k}$. the $k$ th row of matrix $D$ and by $D \cdot \ell$ the $\ell$ th column of $D$.

We define player 1's best-reply correspondence $B_{1}: \Delta^{N} \rightarrow \Delta^{M}$, which assigns to every mixed strategy of player 2 the subset of $\Delta^{M}$ that gives player 1 maximal payoff: $B_{1}(q)=\arg \max _{p \in \Delta^{M}} p^{\top} A q$ for all $q \in \Delta^{N}$. Similarly, player 2's best-reply correspondence $B_{2}: \Delta^{M} \rightarrow \Delta^{N}$ is defined by $B_{2}(p)=\arg \max _{q \in \Delta^{N}} p^{\top} B q$ for all $p \in \Delta^{M}$. A strategy combination $\left(p^{*}, q^{*}\right) \in \Delta^{M} \times \Delta^{N}$ is called a Nash equilibrium if $p^{*} \in B_{1}\left(q^{*}\right)$ and $q^{*} \in B_{2}\left(p^{*}\right)$. We denote the set of all Nash equilibria of a game $(A, B)$ by $\mathrm{NE}(A, B)$. A fundamental result in game theory (Nash (1951)) states that for every $A, B \in \mathbb{R}^{M \times N}$ the set $\mathrm{NE}(A, B)$ is nonempty.

\section{Correlated Equilibria in Bimatrix Games}

A correlated strategy is a probability distribution $\Pi \in \Delta^{M \times N}$ over the set of all pure strategy profiles, i.e., a matrix $\Pi \in \mathbb{R}^{M \times N}$ that satisfies

$$
\forall_{i \in M, j \in N}: \Pi_{i j} \geq 0
$$

and

$$
\sum_{(i, j) \in M \times N} \Pi_{i j}=1 .
$$

A correlated strategy is implemented in the following way:

(1) An arbiter, who is not a player himself, draws an element $x=(i, j) \in M \times N$ according to the probability distribution $\Pi$.

(2) The arbiter tells player 1 which row $i$ has been drawn and player 2 which column $j$ has been drawn.

(3) Knowing the distribution $\Pi$ and knowing the information the arbiter has given him about the realisation of $x$, each player chooses a (mixed) strategy from his strategy space.

(4) The payoff to each player is determined by his expected payoff, as in the mixed extension of the game. 
A correlated strategy $\Pi$ is a correlated equilibrium if in the third stage, the expected payoff of player 1 is maximal when he plays row $i$ with probability 1 for all possible realisations of $i$ :

$$
\forall_{i, k \in M}: \Pi_{i} \cdot A_{i}^{\top} \geq \Pi_{i} \cdot A_{k}^{\top}
$$

and similarly, if the expected payoff of player 2 is maximal when he plays column $j$ with probability 1 for all realisations of $j$ :

$$
\forall_{j, \ell \in N}: \Pi_{\cdot j}^{\top} B_{\cdot j} \geq \Pi_{\cdot j}^{\top} B \cdot \ell .
$$

In other words, a correlated equilibrium is a correlated strategy in which for each realisation of the random draw by the arbiter, playing the row or column the arbiter "advises" is incentive compatible for both players. We denote the set of correlated equilibria of a game $(A, B)$ by $\operatorname{CE}(A, B) \subseteq \Delta^{M \times N}$.

The set of correlated equilibria is the solution set of the system of linear inequalities (3.1)-(3.4). Hence, it is a convex polytope in the $(|M \times N|-1)$-dimensional space $\Delta^{M \times N}$. Lemma 3.1, which follows from Lemma 1 on page 197 in Moulin (1986), shows that Nash equilibria "are" in fact correlated equilibria.

Lemma 3.1. Let $p \in \Delta^{M}, q \in \Delta^{N}$. Then $(p, q) \in \mathrm{NE}(A, B)$ if and only if $p q^{\top} \in$ $\mathrm{CE}(A, B)$.

Proof. Define $\Pi=p q^{\top}$. By construction, $\Pi$ satisfies (3.1) and (3.2). Next, let $i, k \in M$. Substituting $\Pi_{i \ell}=p_{i} q_{\ell}$ and $\Pi_{k \ell}=p_{k} q_{\ell}$, (3.3) can be rewritten as $p_{i} A_{i} . q \geq p_{i} A_{k} . q$, which holds if and only if $p_{i}=0$ or $A_{i} . q \geq A_{k} . q$. Because $k$ is chosen arbitrarily, we find that the incentive compatibility constraints corresponding to row $i$ are satisfied if and only if this row is played with probability zero whenever it is not a pure best response to $q$. Hence, (3.3) is equivalent to $p \in B_{1}(q)$. Similarly, (3.4) holds if and only if $q \in B_{2}(p)$. From this the assertion follows.

We know that $\operatorname{NE}(A, B)$ need not be convex, so there are matrices $A$ and $B$ such that the set of correlated equilibria, as being a convex polytope containing the set of Nash equilibria, is strictly larger than the set of Nash equilibria. Therefore, the concept of correlated equilibrium is a coarsening of the concept of Nash equilibrium.

\section{Example}

To illustrate the concepts of correlated equilibrium and projection, we present an example of a $2 \times 2$ bimatrix game, which is taken from Aumann (1974).

The game $(A, B)$, with $|M|=|N|=2$, is determined by the following payoff matrices of player 1 and 2 , respectively:

$$
A=\left[\begin{array}{ll}
6 & 2 \\
7 & 0
\end{array}\right], \quad B=\left[\begin{array}{ll}
6 & 7 \\
2 & 0
\end{array}\right]
$$


This game has two Nash equilibria in pure strategies, namely $((1,0),(0,1))$ and $((0,1),(1,0))$, and one equilibrium in mixed strategies, namely $\left(\left(\frac{2}{3}, \frac{1}{3}\right),\left(\frac{2}{3}, \frac{1}{3}\right)\right)$. The set of correlated equilibria can be found by solving the system of linear inequalities (3.1)-(3.4), which results in:

$$
\mathrm{CE}(A, B)=\mathrm{Conv}\left\{\left[\begin{array}{ll}
0 & 1 \\
0 & 0
\end{array}\right],\left[\begin{array}{ll}
0 & 0 \\
1 & 0
\end{array}\right],\left[\begin{array}{cc}
\frac{4}{9} & \frac{2}{9} \\
\frac{2}{9} & \frac{1}{9}
\end{array}\right],\left[\begin{array}{cc}
\frac{1}{2} & \frac{1}{4} \\
\frac{1}{4} & 0
\end{array}\right],\left[\begin{array}{cc}
0 & \frac{2}{5} \\
\frac{2}{5} & \frac{1}{5}
\end{array}\right]\right\} .
$$

The first three extreme points of $\mathrm{CE}(A, B)$ correspond to the Nash equilibria, while the two other ones are projections of the completely mixed equilibrium. This example illustrates two known results concerning the extreme points of $\mathrm{CE}(A, B)$ : every extreme point of a maximal Nash set is also an extreme point of the set of correlated equilibria (Evangelista and Raghavan (1996) and Canovas et al. (1999)) and in $2 \times 2$ bimatrix games, $\operatorname{CE}(A, B) \neq \operatorname{Conv}\{\mathrm{NE}(A, B)\}$ if and only if $|\mathrm{NE}(A, B)|=3$ (Peeters and Potters (1999)).

The payoff profiles that correspond to the three Nash equilibria are $(2,7),(7,2)$ and $\left(\frac{14}{3}, \frac{14}{3}\right)$, whereas the fourth extreme point of $\mathrm{CE}(A, B)$ has a payoff profile of $\left(\frac{21}{4}, \frac{21}{4}\right)$. We conclude that, in this example, players can improve upon the efficient payoff profiles that result from the Nash equilibria by correlating their actions.

In Fig. 1, we depict the set of correlated equilibria in $\Delta^{M \times N}$, which is a tetrahedron. The three extreme points of $\operatorname{CE}(A, B)$ that correspond to the Nash equilibria are represented by bullets. The two pure equilibria are corner points of the tetrahedron, i.e., extreme points of $\Delta^{M \times N}$, while the completely mixed equilibrium lies in the interior. The two other extreme points of $\mathrm{CE}(A, B)$ are represented by circles and lie on the faces containing both pure Nash equilibria.

The two extreme points of $\mathrm{CE}(A, B)$ that do not correspond to the Nash equilibria are projections of the completely mixed equilibrium. The extreme point on the back face in Fig. 1 lies on the line through the front corner of the tetrahedron and

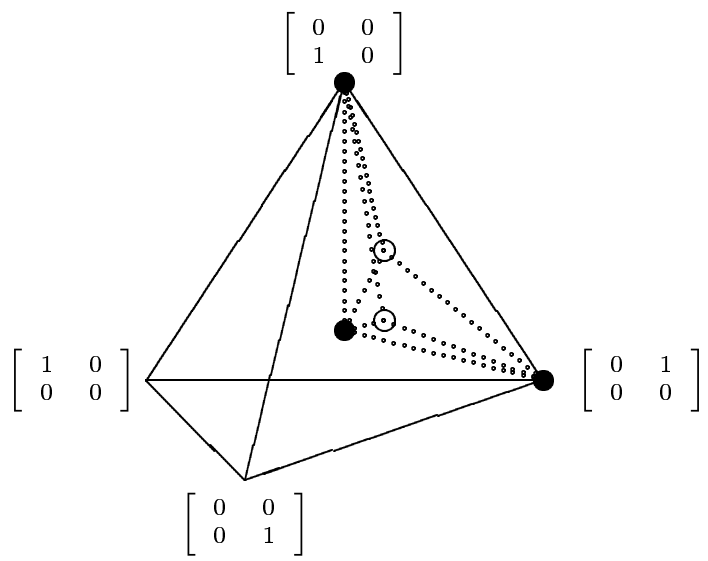

Fig. 1. Correlated equilibria in $\Delta^{M \times N}$. 
the completely mixed equilibrium. Similarly, the extreme point on the front-right face lies on the line through the left corner and the completely mixed equilibrium. Such a projection onto a face of the tetrahedron is the geometric equivalent of the Bayesian updating that was discussed in Sec. 1.

\section{Projection}

In this section we establish under which circumstances we can obtain a correlated equilibrium by projecting a Nash equilibrium ("creating zeroes"). Our main result will be the Projection Theorem, which gives necessary and sufficient conditions for creating a zero. In addition, we will prove some lemmas to further clarify this theorem and to extend it towards creating multiple zeroes.

We start with the Projection Theorem.

Theorem 5.1 (Projection Theorem). Let $(p, q) \in \mathrm{NE}(A, B)$ be such that $\left|C^{M}(p)\right|>1$ and $\left|C^{N}(q)\right|>1$ and let $\left(i_{0}, j_{0}\right) \in C^{M}(p) \times C^{N}(q)$. Then $\left(p q^{\top}\right)^{-\left\{\left(i_{0}, j_{0}\right)\right\}} \in \mathrm{CE}(A, B)$ if and only if $e^{i_{0}, M} \in B_{1}\left(q^{-\left\{j_{0}\right\}}\right)$ and $e^{j_{0}, N} \in$ $B_{2}\left(p^{-\left\{i_{0}\right\}}\right)$.

Proof. Define $\Pi=p q^{\top}, \Pi^{\prime}=\Pi^{-\left\{\left(i_{0}, j_{0}\right)\right\}}$ and $\alpha=\left(1-\Pi_{i_{0} j_{0}}\right)^{-1}$.

" $\Leftarrow$ " Assume $e^{i_{0}, M} \in B_{1}\left(q^{-\left\{j_{0}\right\}}\right)$ and $e^{j_{0}, N} \in B_{2}\left(p^{-\left\{i_{0}\right\}}\right)$. By construction, $\Pi^{\prime}$ satisfies (3.1) and (3.2). Let $i \in M$ and $k \in M \backslash\{i\}$. We check (3.3) by distinguishing between two cases.

(1) Suppose $i=i_{0}$. We have $\Pi_{i}^{\prime}\left(A_{i} \text {. }-A_{k} .\right)^{\top}=\sum_{\ell \in C^{N}(q) \backslash\left\{j_{0}\right\}} \Pi_{i \ell}^{\prime}\left(A_{i \ell}-A_{k \ell}\right)=$ $\alpha \sum_{\ell \in C^{N}(q) \backslash\left\{j_{0}\right\}} \Pi_{i \ell}\left(A_{i \ell}-A_{k \ell}\right)=\alpha p_{i} \sum_{\ell \in C^{N}(q) \backslash\left\{j_{0}\right\}} q_{\ell}\left(A_{i \ell}-A_{k \ell}\right) \geq 0$, where the inequality follows from $e^{i_{0}, M} \in B_{1}\left(q^{-\left\{j_{0}\right\}}\right)$.

(2) Suppose $i \neq i_{0}$. $\Pi_{i .}^{\prime}\left(A_{i} \text {. }-A_{k} \text {. }\right)^{\top}=\alpha \Pi_{i} .\left(A_{i} \text {. }-A_{k} \text {. }\right)^{\top} \geq 0$, because $(p, q) \in$ $\mathrm{NE}(A, B)$.

From (1) and (2) we conclude that for all $i, k \in M$ we have $\Pi_{i}^{\prime} . A_{i}^{\top} \geq \Pi_{i}^{\prime} . A_{k}^{\top}$, so the incentive compatibility constraints of player $1,(3.3)$, are satisfied. Analogously, by interchanging the roles of the players, player 2's incentive compatibility constraints, (3.4), are satisfied as well. Hence, $\Pi^{\prime} \in \mathrm{CE}(A, B)$.

" $\Rightarrow$ " Assume $\Pi^{\prime} \in \mathrm{CE}(A, B)$. Then for all $k \in M \backslash\left\{i_{0}\right\}$ we have $\Pi_{i_{0}}^{\prime} .\left(A_{i_{0}}\right.$. $\left.A_{k}.\right)^{\top} \geq 0$, which is equivalent to $\alpha p_{i_{0}} \sum_{\ell \in C^{N}(q) \backslash\left\{j_{0}\right\}} q_{\ell}\left(A_{i_{0} \ell}-A_{k \ell}\right) \geq 0$. Because $\alpha p_{i_{0}}>0$, this implies $e^{i_{0}, M} \in B_{1}\left(q^{-\left\{j_{0}\right\}}\right)$. In a similar manner, $e^{j_{0}, N} \in B_{2}\left(p^{-\left\{i_{0}\right\}}\right)$ is also satisfied.

Remark 5.1. If $(p, q)$ is a completely mixed equilibrium, that is $(p, q) \in$ $\mathrm{NE}^{\circ}(A, B)=\left\{(p, q) \in \mathrm{NE}(A, B) \mid C^{M}(p)=M, C^{N}(q)=N\right\}$, then $\Pi_{i \cdot}\left(A_{i}-A_{k}\right)^{\top}=$ 0 for all $i, k \in M$. In this case, it follows from (1) of the " $\Leftarrow$ " part of the proof that $\Pi^{\prime}-\Pi$ satisfies the incentive compatibility constraints (3.3) and (3.4). This means that the projected correlated equilibrium is in a sense "more" incentive compatible than the original Nash equilibrium. 
Theorem 5.1 gives necessary and sufficient conditions for creating a zero in terms of best-replies. In order to reformulate these conditions, we need to introduce the notion of carrier-restricted best-reply correspondence. For all $p \in \Delta^{M}$ and $q \in \Delta^{N}$, we define $B_{1}^{*}(q ; p)=\arg \max _{p^{\prime} \in \Delta^{M}, C^{M}\left(p^{\prime}\right) \subseteq C^{M}(p)}\left(p^{\prime}\right)^{\top} A q$ to be the carrier-restricted best-reply of player 1 against $q$, where he is only allowed to use those pure strategies that are played with positive probability according to $p . B_{2}^{*}(p ; q)$ is defined in a similar manner.

The next lemma states that the conditions $e^{i_{0}, M} \in B_{1}\left(q^{-\left\{j_{0}\right\}}\right)$ and $e^{j_{0}, N} \in$ $B_{2}\left(p^{-\left\{i_{0}\right\}}\right)$ in Theorem 5.1 are satisfied if and only if the pure strategy combination $\left(i_{0}, j_{0}\right)$ consists of "mutually worst responses", i.e., $i_{0}$ gives player 1 the lowest possible payoff against $j_{0}$ within the carrier of $p$ and $j_{0}$ gives player 2 the lowest possible payoff against $i_{0}$ within the carrier of $q$.

Lemma 5.1. Let $(p, q) \in \mathrm{NE}(A, B)$ with $\left|C^{N}(q)\right|>1$ and let $(i, j) \in C^{M}(p) \times$ $C^{N}(q)$, then $e^{i, M} \in B_{1}^{*}\left(q^{-\{j\}} ; p\right)$ if and only if $\left(e^{i, M}\right)^{\top} A e^{j, N} \leq\left(e^{k, M}\right)^{\top} A e^{j, N}$ for all $k \in C^{M}(p)$.

Proof. " $\Rightarrow$ " Assume $e^{i, M} \in B_{1}^{*}\left(q^{-\{j\}} ; p\right)$ and suppose there exists a $k \in C^{M}(p)$ such that $\left(e^{i, M}\right)^{\top} A e^{j, N}>\left(e^{k, M}\right)^{\top} A e^{j, N}$. Then $\left(e^{i, M}\right)^{\top} A q=q_{j}\left(e^{i, M}\right)^{\top} A e^{j, N}+(1-$ $\left.q_{j}\right)\left(e^{i, M}\right)^{\top} A q^{-\{j\}}>q_{j}\left(e^{k, M}\right)^{\top} A e^{j, N}+\left(1-q_{j}\right)\left(e^{i, M}\right)^{\top} A q^{-\{j\}} \geq q_{j}\left(e^{k, M}\right)^{\top} A e^{j, N}+$ $\left(1-q_{j}\right)\left(e^{k, M}\right)^{\top} A q^{-\{j\}}=\left(e^{k, M}\right)^{\top} A q$. But then $p \notin B_{1}(q)$, because player 1 is better off playing $e^{k, M}$ with probability zero. Hence, $(p, q) \notin \mathrm{NE}(A, B)$. Contradiction.

" $\Leftarrow "$ Assume $\left(e^{i, M}\right)^{\top} A e^{j, N} \leq\left(e^{k, M}\right)^{\top} A e^{j, N}$ for all $k \in C^{M}(p)$. Define $\alpha=\left(1-q_{j}\right)^{-1}$ and let $k \in C^{M}(p)$. Because $(p, q) \in \mathrm{NE}(A, B)$, we must have that $\left(e^{i, M}\right)^{\top} A q=\left(e^{k, M}\right)^{\top} A q$. Then $\left(e^{i, M}\right)^{\top} A q^{-\{j\}}=\alpha\left(\left(e^{i, M}\right)^{\top} A q-\right.$ $\left.q_{j}\left(e^{i, M}\right)^{\top} A e^{j, N}\right)=\alpha\left(\left(e^{k, M}\right)^{\top} A q-q_{j}\left(e^{i, M}\right)^{\top} A e^{j, N}\right) \geq \alpha\left(\left(e^{k, M}\right)^{\top} A q-\right.$ $\left.q_{j}\left(e^{k, M}\right)^{\top} A e^{j, N}\right)=\left(e^{k, M}\right)^{\top} A q^{-\{j\}}$. Hence, $e^{i, M} \in B_{1}^{*}\left(q^{-\{j\}} ; p\right)$.

Of course, a similar result can be obtained for player 2. Note that Lemma 5.1 need not hold if we take the strategy space $B_{1}\left(q^{-\{j\}}\right)$ instead of the carrier-restricted one $B_{1}^{*}\left(q^{-\{j\}} ; p\right)$. Therefore, from now on we will only consider bimatrix games that possess a completely mixed Nash equilibrium.

We now have easy conditions to check whether creating a zero in a probability matrix representing a Nash equilibrium yields a correlated equilibrium. The next step is to show that if we are allowed to create zeroes at a number of positions separately, creating zeroes at these positions simultaneously yields yet another correlated equilibrium.

Lemma 5.2. Let $(p, q) \in \mathrm{NE}^{\circ}(A, B)$ and let $I \varsubsetneqq M \times N, I \neq \emptyset$. If $\left(p q^{\top}\right)^{-\{u\}} \in$ $\mathrm{CE}(A, B)$ for all $u \in I$, then $\left(p q^{\top}\right)^{-I} \in \mathrm{CE}(A, B)$.

Proof. Assume $\left(p q^{\top}\right)^{-\{u\}} \in \mathrm{CE}(A, B)$ for all $u \in I$. Define $\Pi=p q^{\top}, \Pi^{\prime}=\Pi^{-I}$ and $\alpha=1-\sum_{u \in I} \Pi_{u}$. By construction, $\Pi^{\prime}$ satisfies (3.1) and (3.2). To check (3.3) 
and (3.4), we use the following relation:

$$
\Pi^{\prime}=\alpha^{-1} \sum_{u \in I}\left(1-\Pi_{u}\right)\left(\Pi^{-\{u\}}-\Pi\right)+\Pi .
$$

By Remark 5.1, each of the $\left(\Pi^{-\{u\}}-\Pi\right)$ satisfies (3.3) and (3.4). The probability matrix $\Pi$ corresponds to a Nash equilibrium and therefore also satisfies the incentive compatibility constraints. Because $\Pi^{\prime}$ is a positive linear combination of these matrices, it must satisfy (3.3) and (3.4) as well. Hence, $\Pi^{\prime} \in \operatorname{CE}(A, B)$.

When investigating how to project a particular Nash equilibrium, we have to take the incentive compatibility constraints of both players into account. However, if we create an entire row of zeroes, the incentive compatibility constraint corresponding to that row will be trivially satisfied. Hence, if we want to create an entire row of zeroes, we only have to look at the incentive compatibility constraints of player 2, as the following lemma shows.

Lemma 5.3. Let $(p, q) \in \mathrm{NE}^{\circ}(A, B)$. Let $i \in M$. If $e^{j, N} \in B_{2}\left(p^{-\{i\}}\right)$ for all $j \in N$, then $\left(p^{-\{i\}} q^{\top}\right) \in \mathrm{CE}(A, B)$.

Proof. Assume $e^{j, N} \in B_{2}\left(p^{-\{i\}}\right)$ for all $j \in N$. Obviously, $\left(p^{-\{i\}} q^{\top}\right)$ satisfies (3.1)(3.3). Applying Lemma 5.1, we know that for all $j, \ell \in N$ we have $\left(e^{i, M}\right)^{\top} B e^{j, N}=$ $\left(e^{i, M}\right)^{\top} B e^{\ell, N}$. But then (3.4) follows using the same argument as in the proof of Theorem 5.1. Hence, $\left(p^{-\{i\}} q^{\top}\right) \in \mathrm{CE}(A, B)$.

The conditions in Lemma 5.3 are weaker than the conditions in Lemma 5.2, because the incentive compatibility constraints of one of the players can be left out. Note that it follows from Lemma 3.1 that the resulting correlated equilibrium is a Nash equilibrium.

Lemma 5.2 has some obvious extensions for combinations with entire rows or columns. These can all be proved in a similar manner and are left to the reader.

\section{References}

Aumann, R. (1974). "Subjectivity and correlation in randomized strategies", J. Math. Econ., Vol. 1, 67-95.

Aumann, R. (1987). "Correlated equilibrium as an expression of Bayesian rationality", Econometrica, Vol. 55, 1-18.

Canovas, S., P. Hansen and B. Jaumard (1999). "Nash equilibria from the correlated equilibria viewpoint", Int. Game Theory Review, Vol. 1, 33-44.

Evangelista, F. and T. Raghavan (1996). "A note on correlated equilibrium", Int. J. Game Theory, Vol. 25, 35-42.

Moulin, H. (1986), Game theory for the social sciences. New York: New York University Press.

Nash, J. (1951). "Non-cooperative games", Ann. Mathematics, Vol. 54, 286-295.

Osborne, M. and A. Rubinstein (1994). A Course in Game Theory. Cambridge, MA and London: MIT Press. 
Peeters, R. and J. Potters (1999), "On the structure of the set of correlated equilibria in two-by-two bimatrix games". CentER Discussion Paper 9945, Tilburg University, Tilburg, The Netherlands. 
Copyright $\odot 2002$ EBSCO Publishing 